\title{
Stable phase CdS nanoparticles for optoelectronics: A study on surface morphology, structural and optical characterization
}

\author{
SURESH KUMAR $^{1 *}$, J.K. SHARMA ${ }^{2}$ \\ ${ }^{1}$ Department of Applied Sciences and Humanities, Maharishi Markandeshwar University, Sadopur, \\ Ambala-134007, Haryana, India \\ ${ }^{2}$ Department of Physics, Maharishi Markandeshwar University, Mullana, Ambala-133207, India
}

\begin{abstract}
This work presents a study on the surface morphology, structure and optical behavior of stable phase cadmium sulphide (CdS) nanoparticles synthesized via co-precipitation technique. Scanning electron microscopy (SEM) analysis has been employed to study a cluster formation in the aggregated nanoparticles. An image analysis approach using ImageJ has been used to measure the size of nanoparticles from the SEM micrographs. Fourier transform infrared spectroscopic (FT-IR) analysis identified absorption peaks of $\mathrm{Cd}-\mathrm{S}$ stretching along with moisture content. X-ray diffraction (XRD) analysis showed that $\mathrm{CdS}$ nanoparticles crystallized in wurtzite structure with a preferential orientation along $\left(\begin{array}{lll}0 & 0 & 2\end{array}\right)$ plane. The particle size, microstrain and lattice constants have been evaluated using XRD data. The lattice parameters of these nanoparticles were found to be shorter than the bulk value which led to lattice contraction. The optical absorption study showed a blue shift in the fundamental absorption edge indicating a quantum size effect.
\end{abstract}

Keywords: CdS nanoparticles; surface morphology; structural analysis; optical absorption; quantum size effect

(c) Wroclaw University of Technology.

\section{Introduction}

Recent developments in nanotechnology proved that many novel devices of the future will be based on the properties of nanomaterials [1-4]. Presently, nanomaterials are produced in various nanoforms, such as quantum dots, nanoparticles, nanowires, nanotubes, nanoprisms, nanofilms etc., by employing top-down and bottom-up approaches $[4,5]$. The nanoforms of semiconductor materials have received great attention due to their unique properties and potential applications. They exhibit entirely different physicochemical properties than their bulk counterparts, due to increased relative surface area and quantum confinement $[4,6]$. The particle size and morphology of these nanomaterials can be easily changed and hence, enhancement in their various properties, such as reactivity, strength, optoelectronic,

*E-mail: sureshlakhanpal@gmail.com electrical, magnetic behavior etc., and finally their potential applications, can be achieved. Binary semiconductors (group II - VI) in various nanoforms have many scientific and technological applications [7-9]. CdS is a promising binary II VI semiconductor used in variety of optoelectronic applications, including solar cell windows, laser communication, photoconductors, field effect transistors, sensors, photocatalysts, biological indicators, light emitting diodes, etc. [9-12]. It is a wide band gap semiconductor lightly soluble in water and highly soluble in acid. In nature, it is found in greenockite (wurtzite), hawleyite (zinc blende) and rocksalt mineral structures [6]. The zinc blende to wurtzite transformation involves a change in symmetry whereas wurtzite to rocksalt transformation involves not only a change in symmetry but also a change in the nearest neighbor atomic coordination from four to six [6]. The wurtzite $(\alpha-\mathrm{CdS})$ and zinc blende $(\beta-\mathrm{CdS})$ forms are the most common crystal formation 
of CdS having lattice parameters $\mathrm{a}_{\mathrm{wz}}=4.137 \AA$, $\mathrm{c}_{\mathrm{wZ}}=6.7144 \AA$ and $\mathrm{a}_{\mathrm{zb}}=5.832 \AA$, respectively [13]. The wurtzite form of $\mathrm{CdS}$, due to its stable nature, has been preferred for the fabrication of devices. Therefore, more extensive studies on the nanoforms of $\mathrm{CdS}$ semiconductor and their various properties have been carried out. A variety of chemical routes to synthesize $\mathrm{CdS}$ nanoforms have been employed [6, 9, 14-22]. However, the co-precipitation technique is an important bottomup approach for the synthesis of nanoparticles. It is simple, inexpensive, free from complex instrument handling and a low temperature processing technique [23]. It also has the potential advantage to prepare nanoparticles of relatively low cost, high purity and controlled morphology. It can be performed using a wide range of precursors with easy control over synthesis parameters, such as $\mathrm{pH}$, temperature, time, stirring rate, concentration of reactants etc. The controlled variation of the synthesis parameters may lead to optimizing the size, geometry and morphology of the resulting nanoparticles.

This work reports the synthesis of CdS nanoparticles via co-precipitation technique without any capping agent. The present study is an effort to explore the physical and optical behavior of chemically synthesized stable phase CdS nanoparticles.

\section{Experimental}

\subsection{Synthesis of CdS nanoparticles}

All chemicals used in the synthesis were analytical grade products purchased from Merck, India and were used as received without further purification. The chemicals that were used for the synthesis of $\mathrm{CdS}$ nanoparticles were cadmium chloride anhydrous $\left(\mathrm{CdCl}_{2} \mathrm{H}_{2} \mathrm{O}\right)$ as the source of $\mathrm{Cd}^{2+}$ ions, thiourea $\left(\mathrm{CS}\left(\mathrm{NH}_{2}\right)_{2}\right)$ as the source of $\mathrm{S}^{2-}$ ions and ammonia solution $\left(\mathrm{NH}_{4} \mathrm{OH}\right)$ as the complexing agent to control precipitation. To prepare $\mathrm{CdS}$ nanoparticles, the equimolar $(0.2 \mathrm{M})$ aqueous solutions of the reactants, cadmium chloride and thiourea were prepared using double distilled water (Millipore, $15 \mathrm{M} \Omega \cdot \mathrm{cm}$ ) separately.
The ammonia solution ( $25 \%$ ) was added dropwise through a burette into $25 \mathrm{~mL}$ of cadmium chloride solution under slow stirring. It was added to dissolve the white precipitate of $\mathrm{Cd}(\mathrm{OH})_{2}$ under constant magnetic stirring rate $(300 \mathrm{rpm})$ till a clear transparent solution formed. Finally $25 \mathrm{~mL}$ aqueous solution of thiourea was mixed into it. The $\mathrm{pH}$ of the solution was stabilized at $10.5 \pm 0.1$ by ammonia addition. The whole synthesis process was carried out under continuous stirring at temperature of $70 \pm 2{ }^{\circ} \mathrm{C}$ using a hot plate magnetic stirrer. Initially, as the reaction started the solution color gradually changed from transparent to light yellow, which suggested precipitation, and finally turned to dark yellow. To remove any type of unreacted chemicals or impurities, the precipitates were washed several times with double distilled water and finally collected by centrifugation process. The collected $\mathrm{CdS}$ precipitate was first dried in a glass desiccator to get CdS nanoparticles in powder form and then annealed in a muffle furnace at temperature of $300{ }^{\circ} \mathrm{C}$ for 3 hours.

\subsection{Characterization techniques}

The surface morphology of CdS nanoparticles has been studied using SEM (JEOL JSM- 6100) operated at $20 \mathrm{kV}$ at a magnification of 14000 . An image processing approach using ImageJ programme has been also used to measure the particle size from 2D SEM images. Fourier transform infrared spectroscopy (FT-IR) measurement has been performed by Perkin Elmer 1600 FT-IR in the spectral range of $400 \mathrm{~cm}^{-1}$ to $4000 \mathrm{~cm}^{-1}$ in $\% \mathrm{~T}$ mode using $\mathrm{KBr}$ pellet technique. The $\mathrm{X}$-ray powder diffraction (XRD) data was recorded using PANaytical's X'Pert-Pro diffractometer, with $\mathrm{CuK} \alpha$ radiation $(\lambda=1.5406 \AA)$, operating at $40 \mathrm{kV}$ and $40 \mathrm{~mA}$. The $2 \theta$ scanning range was from $10^{\circ}$ to $60^{\circ}$ in steps of $0.02^{\circ}$. The XRD data was further analyzed for baseline correction, fitting and peak parameters using Fityk-093 software. The optical absorption spectrum (210 nm to $1000 \mathrm{~nm}$ ) was recorded using an UV-Vis-NIR Perkin Elmer Lambda 40 spectrophotometer. 


\section{Results and discussion}

\subsection{Surface morphology analysis}

Scanning electron microscopy (SEM) micrograph (Fig. 1a) shows that $\mathrm{CdS}$ nanoparticles exist in flocks. These flocks are formed by infinite numbers of crystallites which aggregated densely. The formation of these compact flocks in CdS powder is due to homogeneous precipitation that occurs during the synthesis process followed by cluster growth of crystallites $[9,18]$. These nanocrystallites are embedded in these clusters and acquire flake like shapes. In case of powder samples the shape, size and boundaries of particles are difficult to identify only using SEM images and hence, ImageJ programme was used for image analysis [24, 25]. Fig. 1b to Fig. 1d show 2D images transformed by following the image analysis procedure within ImageJ programme. The analysis of the SEM image was carried out in different steps such as setting a scale for 2D SEM image, removing noise using Gaussian blur filter, setting threshold (Fig. 1b) and detecting boundaries of the nanoparticles through binary image (Fig. 1c). Finally the binary image was subjected to automatic identification and counting the number of particles (Fig. 1d). The average size of nanoparticles estimated by the imageJ programme through the analyzing tool was found to be $16.3 \mathrm{~nm}$.

\subsection{Fourier transform infrared analysis}

Analyzing the FT-IR spectrum (Fig. 2), it has been observed that many absorption peaks and bands exist in the synthesized CdS nanopowder (inset in Fig. 2) which belong to the molecular alignment of the elements. Normally $\mathrm{Cd}-\mathrm{S}$ stretching frequency has been found in the lower wave number region, i.e. below $700 \mathrm{~cm}^{-1}$ [15]. In our $\mathrm{CdS}$ nanoparticles, a medium strong absorption peak at $651.62 \mathrm{~cm}^{-1}$ belonging to $\mathrm{Cd}-\mathrm{S}$ stretching has been observed. The absorption peaks at $1074.86 \mathrm{~cm}^{-1}$ and $1165.35 \mathrm{~cm}^{-1}$ belong to $\mathrm{C}-\mathrm{N}$ stretch/C $=\mathrm{S}$ of thiourea and the peak at $1373.13 \mathrm{~cm}^{-1}$ corresponds to tris-amine $\mathrm{C}-\mathrm{N}$ stretch shared with $\mathrm{C}-\mathrm{O}$ stretching. Moreover, the peaks at $2000.37 \mathrm{~cm}^{-1}$ and $2150.47 \mathrm{~cm}^{-1}$

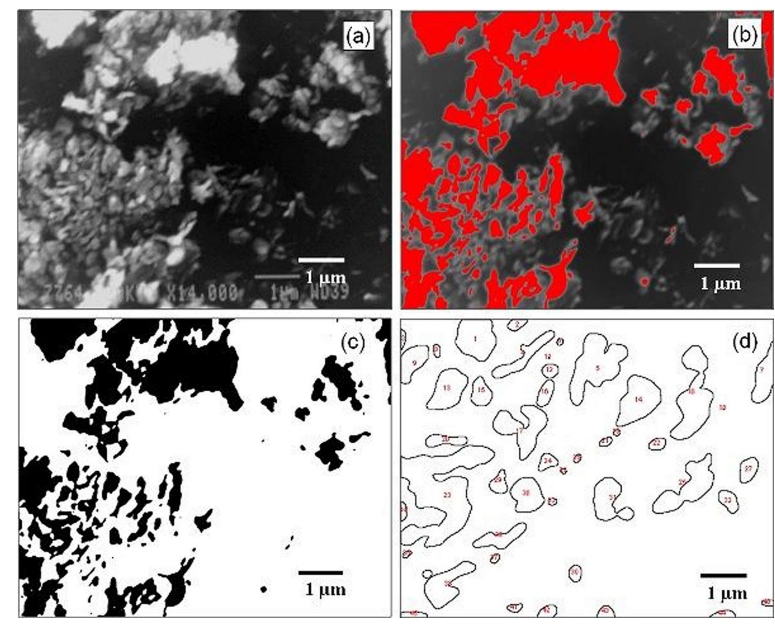

Fig. 1. (a) original 2D SEM micrographs, ImageJ processed images (b) threshold image after eliminating noise (c) binary image for boundary distribution and (d) final image employed to measure particle size.

also correspond to $\mathrm{N}=\mathrm{C}$ stretch or isothiocynate (-NCS) which formed due to hydrolysis of thiourea during the synthesis $[17,26]$. A weak doublet at $2923.61 \mathrm{~cm}^{-1}$ and $2853.01 \mathrm{~cm}^{-1}$ has been assigned to asymmetrical and symmetrical vibrations of $\mathrm{CH}$ group, respectively, while the asymmetric scissor deformation vibration exits at $1445.50 \mathrm{~cm}^{-1}$ [26]. The medium strong and broad absorption bands at $3428.98 \mathrm{~cm}^{-1}$ and $1629.96 \mathrm{~cm}^{-1}$ have been assigned to $\mathrm{O}-\mathrm{H}$ stretching vibration of $\mathrm{H}_{2} \mathrm{O}$ molecules [26]. Another weak absorption peak at $898.46 \mathrm{~cm}^{-1}$ belongs to O-H out of plane bending vibration of $\mathrm{H}_{2} \mathrm{O}$ molecules. These vibrations have been observed because of the moisture content that exists in CdS nanoparticles even after thermal annealing. The existence of respective absorption peaks and bands in FT-IR spectrum confirms the presence of $\mathrm{CdS}$ molecules, the impurities traces, because of chemical reaction of different precursors used, and water molecules or hydroxide ions in the prepared nanoparticles.

\subsection{Structural characterization by X-ray diffraction}

The presence of multiple diffraction peaks with the broad peak profile in the XRD spectrum (Fig. 3) can be attributed to the polycrystalline behavior 


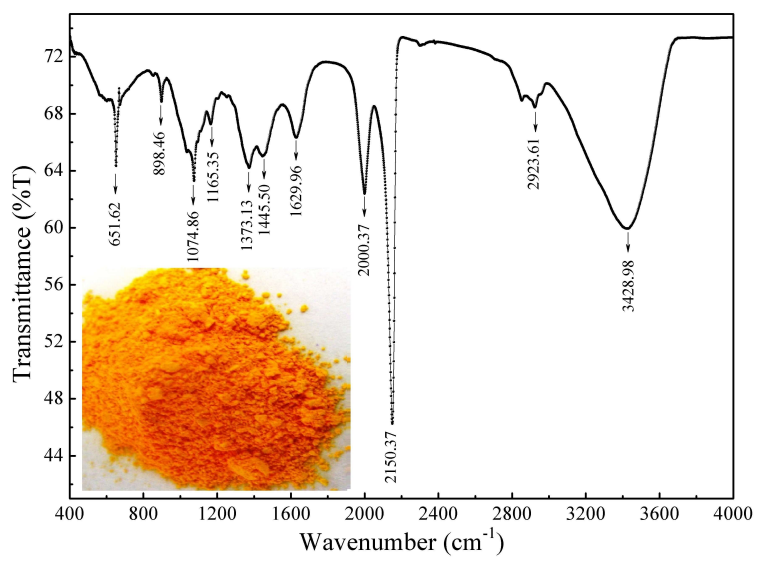

Fig. 2. FT-IR spectrum for CdS nanoparticles; the inset shows original CdS nanopowder.

of the synthesized CdS nanoparticles. Moreover, the diffused background on the XRD spectrum may be due to the amorphous character associated with disordered periodicity of lattice elements. This type of behavior is commonly observed in nanosized particles [14]. The comparison of XRD peak profile and the $2 \theta$ position of diffraction peaks (Table 1) with standard ASTM data confirms the wurtzite $(\alpha-\mathrm{CdS})$ crystal structure with the preferential orientation along (lllll 00 2) plane [10]. An additional peak at $2 \theta=31.33^{\circ}$ has also been detected which corresponds to hcp $\mathrm{CdO}$ [27]. This may point out that the synthesized $\mathrm{CdS}$ nanoparticles are rich in $\mathrm{Cd}$ content. The intensity of diffraction peaks (Table 1) has been observed to be low as compared to bulk CdS [10]. It is also observed that the positions of diffraction peaks for $\mathrm{CdS}$ nanoparticles shift to higher diffraction angles in comparison to bulk CdS. This may be due to lattice contraction which is expected to occur because of higher surface to volume ratio of nanoparticles [18]. The interplanar spacing ' $\mathrm{d}_{\mathrm{hkl}}$ ' values have been evaluated (Table 1) using Bragg's diffraction equation [9]. The smaller values of $d_{h k l}$ than the standard $d_{h k l}$ value also support the lattice contraction in the synthesized CdS nanoparticles. Williamson-Hall (W-H) plot (Fig. 4) has been used to evaluate the particle size $\left(\mathrm{D}_{\mathrm{v}}\right)$ and microstrain $\left(\varepsilon_{\mathrm{hkl}}\right)$ as [28]:

$$
\beta_{h k l} \cos \theta_{h k l}=\left(\frac{\lambda}{D_{v}}\right)+4 \varepsilon_{h k l} \sin \theta_{h k l}
$$

where $\beta_{\mathrm{hkl}}$ is the integral peak width associated with Bragg angle $\left(\theta_{\mathrm{hkl}}\right)$. The obtained values for $\mathrm{D}_{v}$ and $\varepsilon_{\mathrm{hkl}}$ are $15.23 \mathrm{~nm}$ and $6.93 \times 10^{-3}$, respectively. The particle size calculated from XRD analysis is in good agreement with those obtained from imageJ analysis of 2D SEM micrograph. The lattice constants ( $\mathrm{a}$ and $\mathrm{c}$ ) for the hexagonal structure have been determined using the relation [28] as:

$$
\frac{1}{d_{h k l}}=\frac{4}{3}\left\{\frac{h^{2}+h k+k^{2}}{a^{2}}\right\}+\left(\frac{l^{2}}{c^{2}}\right)
$$

where (hkl) are Miller indices of the respective reflecting planes. The average values of a and $\mathrm{c}$ have been calculated as $4.081 \AA$ and $6.662 \AA$ respectively with an error approximation of $\pm 0.001 \AA$. The lattice constants for the synthesized $\mathrm{CdS}$ nanoparticles were found to be less than standard values [10]. Thus it can be concluded that the lattice is contracted which may be due to the presence of defects like surface or interface stress, strain and dislocations, etc., associated with reduced size of nanoparticles [29].

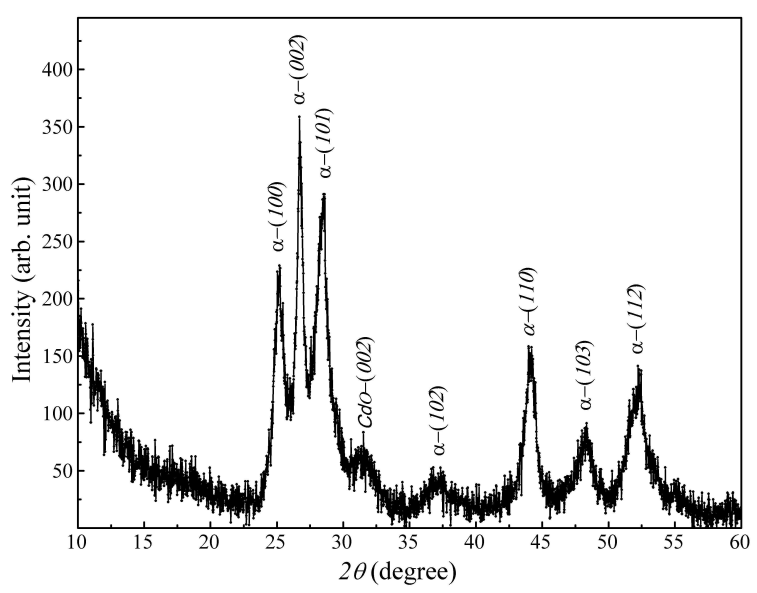

Fig. 3. XRD spectrum for $\mathrm{CdS}$ nanoparticles.

\subsection{Optical characterization}

One of the important features of semiconductor nanoparticles is their optical behavior. Today it is well known that as the particle size of a semiconductor decreases and approaches the size of Bohr exciton the optical band gap increases [9, 30, 31]. This can be observed as a shift in the absorption 
Table 1. Structural parameters for CdS nanoparticles with corresponding standard values.

\begin{tabular}{ccccccc}
\hline \multicolumn{3}{c}{ Observed values } & \multicolumn{3}{c}{ Standard values } & \multirow{2}{*}{ Miller planes } \\
\cline { 1 - 6 } $2 \theta$ [deg.] & $\mathrm{d}_{\text {hkl }}[\AA]$ & Intensity & $2 \theta$ [deg.] & $\mathrm{d}_{\text {hkl }}[\AA]$ & Intensity & \\
\hline \hline 25.17 & 3.535 & 60.72 & 24.85 & 3.582 & 59.21 & $\left(\begin{array}{llll}1 & 0 & 0\end{array}\right)$ \\
26.74 & 3.331 & 100.00 & 26.55 & 3.357 & 42.68 & $\left(\begin{array}{lll}0 & 0 & 2\end{array}\right)$ \\
28.44 & 3.135 & 79.94 & 28.23 & 3.160 & 100.00 & $\left(\begin{array}{llll}1 & 0 & 1\end{array}\right)$ \\
37.10 & 2.421 & 13.75 & 36.68 & 2.439 & 25.83 & $\left(\begin{array}{llll}1 & 0 & 2\end{array}\right)$ \\
44.08 & 2.053 & 43.97 & 43.76 & 2.068 & 45.25 & $\left(\begin{array}{llll}1 & 1 & 0\end{array}\right)$ \\
48.21 & 1.886 & 24.41 & 47.92 & 1.892 & 45.29 & $\left(\begin{array}{llll}1 & 0 & 3\end{array}\right)$ \\
52.18 & 1.751 & 39.21 & 51.92 & 1.761 & 15.27 & $\left(\begin{array}{llll}1 & 1 & 2\end{array}\right)$ \\
\hline
\end{tabular}

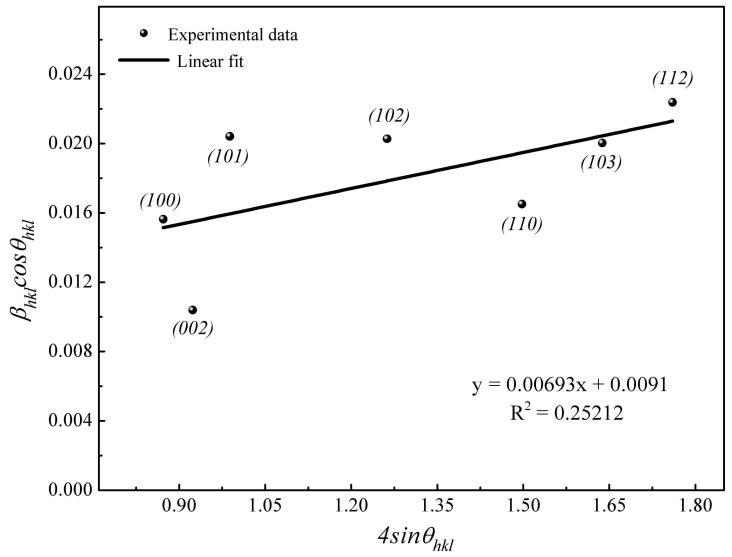

Fig. 4. W-H plot for CdS nanoparticles.

edge toward the lower wavelength side, i.e. blue shift in absorption spectra. The absorption spectrum (Fig. 5) displays a decrease in absorption by $\mathrm{CdS}$ nanoparticles with the increase in wavelength. It has been found that optical absorption is $<20 \%$ in the visible region indicating high transparency of CdS nanoparticles. In various nanoform of semiconductors an excitation peak exists along with absorption edge $[31,32]$ which is estimated by the second derivative of absorption spectrum (Fig. 5). In the present study the excitation peak is found at $290 \mathrm{~nm}$ and for absorption edge at $460 \mathrm{~nm}$. Oliveira et al. [15] has also reported wurtzite $\mathrm{CdS}$ nanoparticles (yellow to orange color) having an absorption edge between $400 \mathrm{~nm}$ to $470 \mathrm{~nm}$. The position of the absorption edge has been observed to be blue shifted, by $53 \mathrm{~nm}$ relative to the fundamental absorption edge of bulk wurtzite CdS (513.6 nm) [6].
This blue shift in the absorption edge is believed to be caused by the quantum confinement effect which is expected in the nano-sized CdS particles [6]. The particle size and optical band gap values for $\mathrm{CdS}$ in various nanoforms have a wide range depending on the synthesis technique. In this study the optical band gap of CdS nanoparticles is calculated to be $2.69 \mathrm{eV}$.

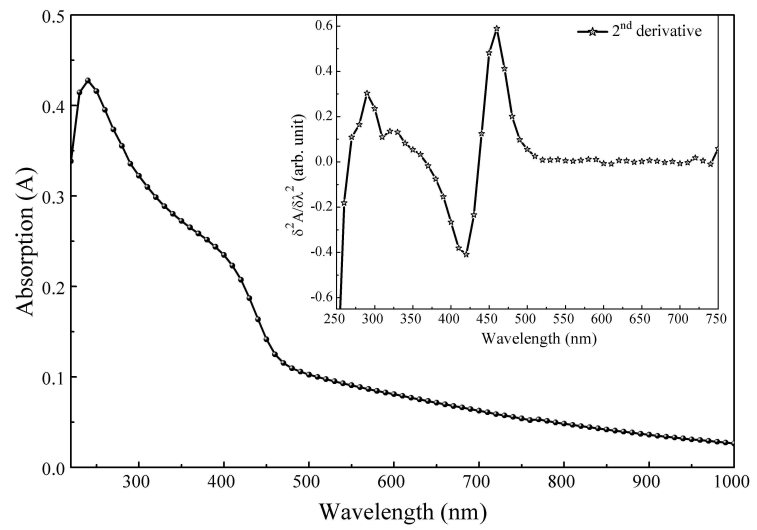

Fig. 5. UV-Vis-NIR absorption spectrum; the inset shows specific energy range second derivative of absorption spectrum.

\section{Conclusion}

In this work we have synthesized orange color CdS nanoparticles (inset Fig. 2) via coprecipitation technique. The homogeneous precipitation resulted in the cluster growth of $\mathrm{CdS}$ nanoparticles. These nanoparticles are polycrystalline in nature having stable (wurtzite) structure with the preferential orientation in $\left(\begin{array}{lll}0 & 0 & 2\end{array}\right)$ plane. 
A $\mathrm{Cd}-\mathrm{S}$ bond stretching has been identified by absorption peak at $651.62 \mathrm{~cm}^{-1}$. Williamson-Hall (W-H) method has been employed to evaluate particle size $(15.23 \mathrm{~nm})$ and microstrain $\left(6.93 \times 10^{-3}\right)$. The particle size, analyzed using ImageJ programme is close to that obtained by W-H method. The lattice constants are found to be slightly less than bulk $\mathrm{CdS}$ values indicating lattice contraction. The existence of excitation peak and blue shift of fundamental absorption edge indicate quantum size effect in the CdS nanoparticles. These stable phase $\mathrm{CdS}$ nanoparticles with enhanced structural and optical characteristics will be more suitable for visible range optoelectronic applications.

\section{Acknowledgements}

The authors acknowledge SAIF, the Panjab University Chandigarh, for providing SEM, FT-IR and XRD facility and the Jaypee University of Information Technology, Solan, (H.P.) for UV-Vis-NIR spectrophotometer facility.

\section{References}

[1] Demming A., Nanotechnology, 22 (2011), 090201.

[2] Kim D.H., LU N., Ghaffari R., Rogers J.A., NPG Asia Mater., 4 (2012), e15.

[3] Chen L., Jiang Y., Wang C., LiU X., Chen Y., JIE J., J. Exp. Nanosci., 5 (2010), 106.

[4] CAO G., Nanostructures \& nanomaterials: synthesis, properties \& applications, Imperial College Press, London, 2004.

[5] Smith A.M., NiE S., Accounts Chem. Res., 16 (2010), 190.

[6] Tai G., Zhou J., Guo W., Nanotechnology, 21 (2010), 175601.

[7] Yao W.T., Yu S.H., Adv. Funct. Mater., 18 (2008), 3357.

[8] Jie J., Zhang W., Bello I., Lee C.S., Lee S.T., Nano Today, 5 (2010), 313.

[9] Kumar S., Sharma P., Sharma V., J. Appl. Phys., 111 (2012), 113510.

[10] Hullavarad N.V., Hullavarad S.S., Karulkar P.C., J. Nanosci. Nanotechno., 8 (2008), 3272.

[11] Zhai T., Fang X., Li L., Bando Y., Golberg D., Nanoscale, 2 (2010), 168.
[12] Li H., WAng X., Xu J., Zhang Q., BAndo Y., GolBerg D., MA Y., Zhai T., Adv. Mater, 11 (2013), 3017.

[13] AMCSD (database code - 0015177 and 0011517).

[14] Chen Q., Bao H., Shen X., Phase Transit., 81 (2008), 591.

[15] Oliveira J.F.A., Milao T.M., Araujo V.D., Moreira M.L., Longo E., Bernardi M.I.B., J. Alloy. Compd., 509 (2011), 6880.

[16] Barote M.A., YAdaV A.A., Masumdar E.U., Physica B, 406 (2011), 1865.

[17] Cabana Z.L., Torres C.M.S., Gonzalez G., Nanoscale Res. Lett., 6 (2011), 523.

[18] Paz M.G.S., Bon R.R., Thin Solid Films, 520 (2011), 999.

[19] Jung D.R., Kim J., Nam S., Nahm C., Choi H., Kim J.I., LeE J., Kim C., PARK B., Appl. Phys. Lett., 99 (2011), 041906.

[20] Chu J., Jin Z., Cai S., Yang J., Hong Z., Thin Solid Films, 520 (2012), 1826.

[21] Mondal B., SAHA S.K., J. Nanopart. Res., 14 (2012), 1049.

[22] LiU S., YAng M.Q., TANG Z.R., XU Y.J., Nanoscale, 7 (2014), 7193.

[23] Kripal R., Gupta A.K., Mishra S.K., Srivastava R.K., PANDEY A.C., PraKash S.G., Spectrochim. Acta A, 76 (2010), 523.

[24] Schneider C.A., Rasband W.S., Eliceiri K.W., Nat. Methods, 9 (2012), 671.

[25] Bhandari K.P., Roland P.J., Kinner T., CaO Y., Choi H., Jeong S., Ellingson R.J., J. Mater. Chem. A, 3 (2015), 853.

[26] Kumar S., Sharma P., Sharma V., IEEE T. Nanotechnol., 13 (2014), 343.

[27] AMCSD (database code - 0012941).

[28] Kale R.B., Lokhande C.D., Semicond. Sci. Tech., 20 (2005), 1.

[29] Kumar S., Sharma P., Sharma V., Electron. Mater. Lett., 9 (2013), 371.

[30] Brus L., J. Phys. Chem., 90 (1986), 2555.

[31] Katsikas L., Eychmilller A., Giersig M., Weller H., Chem. Phys. Lett., 172 (1990), 201.

[32] Acosta M.F., Lerma M.S., Chavez H.A., BarRAZA F.F.C., BON R.R., Solid State Commun., 128 (2003), 407.

Received 2015-10-05 Accepted 2016-01-12 